XXXIX "Jaszowiec" International School and Conference on the Physics of Semiconductors, Krynica-Zdrój 2010

\title{
Built-In Electric Field in High Quality GaN/AlGaN Quantum Wells
}

\author{
K. Zieleniewski, K. PakuŁa, R. Bożek, K. Masztalerz, A. Wysmolek \\ AND R. STĘPNIEWSKI
}

Institute of Experimental Physics, Faculty of Physics, University of Warsaw, Hoża 69, 00-681 Warsaw, Poland

We report studies on electric field built in $\mathrm{GaN} / \mathrm{Al}_{0.09} \mathrm{Ga}_{0.91} \mathrm{~N}$ structure of nominally $6 \mathrm{~nm}$ wide quantum well. The sample was grown in horizontal metal-organic chemical vapor deposition reactor using innovative technology that decreases the density of screw dislocations. Firstly, using visible and mid infra-red interference pattern along the sample, the layer thickness and consequently the quantum well width was determined to vary linearly with the position. Secondly, photoluminescence spectra was taken at different positions. Correlation of those two measurements allows us to determine the built-in electric field to be $0.66 \mathrm{MV} / \mathrm{cm}$, which is considerably larger than previously reported for similar structures.

PACS: 78.55.Cr, 78.67.De, 77.55.hn, 81.15.Gh

\section{Introduction}

The built-in electric field induced by spontaneous and piezoelectric polarization is a fundamental property of GaN $/ \mathrm{Al}_{x} \mathrm{Ga}_{1-x} \mathrm{~N}$ heterostructures [1] both physically and for its practical applications, e.g. in blue lasers, transistors and detectors [2]. We present the results of the photoluminescence (PL) and mid infra-red characterization of $\mathrm{GaN} / \mathrm{Al}_{x} \mathrm{Ga}_{1-x} \mathrm{~N}$ quantum well $(\mathrm{QW})$ structures grown on sapphire with low temperature (LT) AlN nucleation layer. LT AlN layer was grown with extremely low growth rate resulting in better structural ordering [3]. Subsequently grown GaN layer exhibit significant reduction of the internal strain and concentration of screw dislocations, which had been expected to positively influence the quality of the interfaces between sublayers.

\section{Samples}

The set of $\mathrm{GaN} / \mathrm{Al}_{x} \mathrm{Ga}_{1-x} \mathrm{~N}$ QW structures (Fig. 1) were grown along $c$-axis on sapphire substrates in a horizontal, low pressure metal-organic vapor phase epitaxy (MOVPE) reactor. The growth was initiated by the $20 \mathrm{~nm}$ buffer layer consisting of low temperature AlN [3].

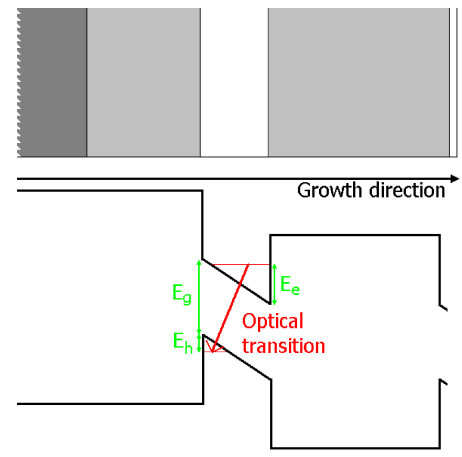

Fig. 1. Nominal physical and electronic layout of the sample.
This was followed by $600 \mathrm{~nm}$ of $\mathrm{Al}_{x} \mathrm{Ga}_{1-x} \mathrm{~N}$ and next $600 \mathrm{~nm}$ barrier layer with Si doping. Then came $10 \mathrm{~nm}$ spacer barrier, $6 \mathrm{~nm}$ QW and finally $20 \mathrm{~nm}$ barrier covered by the $0.5 \mathrm{~nm}$ cap of GaN. The $\mathrm{Al}$ concentration was set constant in the barriers, and for different sample it varied in the range between 0.06 and 0.11 . In this report we concentrate our attention on the results obtained for the sample with $x=0.09$. For this sample we observe constant PL energy of the barrier, indicating constant $\mathrm{Al}$ concentration, whereas QW PL changes almost linearly as a function of position.

\section{Width of the QW}

Interference patterns, observed in room temperature reflectivity measurements in visible range (for 510, 580 and $650 \mathrm{~nm}$, cf. Fig. 2) and mid infrared (Fig. 3), clearly show that the thickness of whole epitaxial layer changes

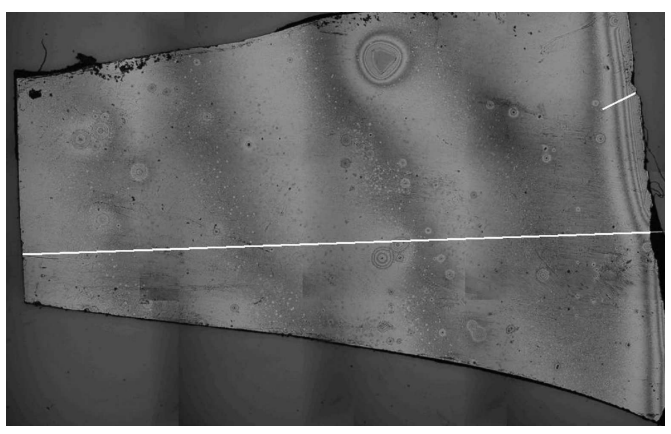

Fig. 2. Example of visible light (here $510 \mathrm{~nm}$ ) interference measurement. This photo is a mosaic of 12 photos. Dark stripes were created due to non-uniform illumination and they should not be confused with actual interference pattern, which lie at some angle. The white lines represent paths on which sample width was measured. The upper one, taken at the edge, was for help in deciding numbers of fringes visible (cf. inset in Fig. 6). Similar images were taken also with 580 and $650 \mathrm{~nm}$ filters. 


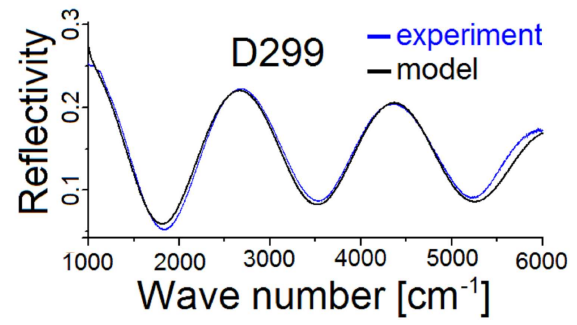

Fig. 3. Medium infra-red reflectivity spectra obtained on the whole sample with the Fourier transform spectroscopy. Analogous spectra have been measured on 5 pieces of the sample (2 mm wide each) and they constitute source for points in Fig. 4.

linearly with respect to position (Fig. 4), which is typical for the type of metal-organic chemical vapor deposition (MOCVD) reactor we used. At a given position of the sample the growth rate should be constant across the structure, so the QW width should be constant fraction of the local sample thickness. This enables us to determine the actual QW width for any position on the sample. These properties of the sample allow us to perform detailed studies of the PL dependence on the QW width $L$.

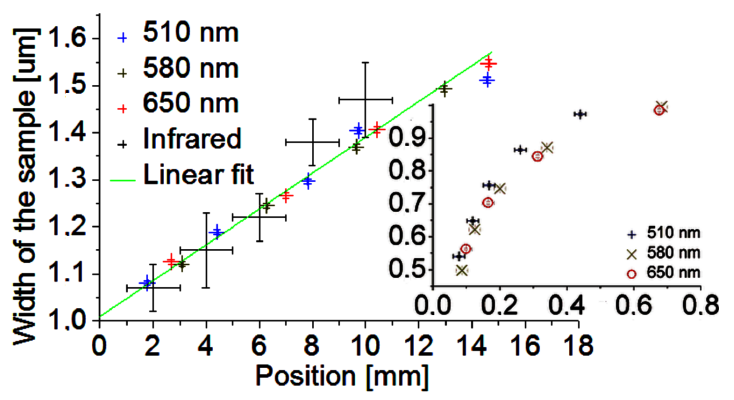

Fig. 4. Measured widths of the sample at different positions support hypothesis that sample width changes linearly with respect to position (fitted line of the form $L=a x+b, a=38.1(17) \mathrm{nm} / \mathrm{mm}, b=1009(14) \mathrm{nm})$. Different growth rates come from different reagents contents; in this case growth rate is limited by availability of gallium. The inset shows non-linear edge width (in $\mu \mathrm{m}$ ) vs. position on the sample (in $\mathrm{mm}$ ) along the upper line in Fig. 2.

\section{Photoluminescence}

Photoluminescence spectra (Fig. 5) were collected at $4.2 \mathrm{~K}$ for different positions on the sample. The observed PL lines are relatively sharp (mean half width at half maximum of $24.8 \mathrm{meV}$, see inset in Fig. 6) in comparison to already published data [4-7]. The observed barrier luminescence shows nearly constant energy across the sample, which supports constant Al concentration assumption. In the simplest approximation the PL energy $E_{\mathrm{PL}}$ depends on electric field $F$ and QW width $L$ as

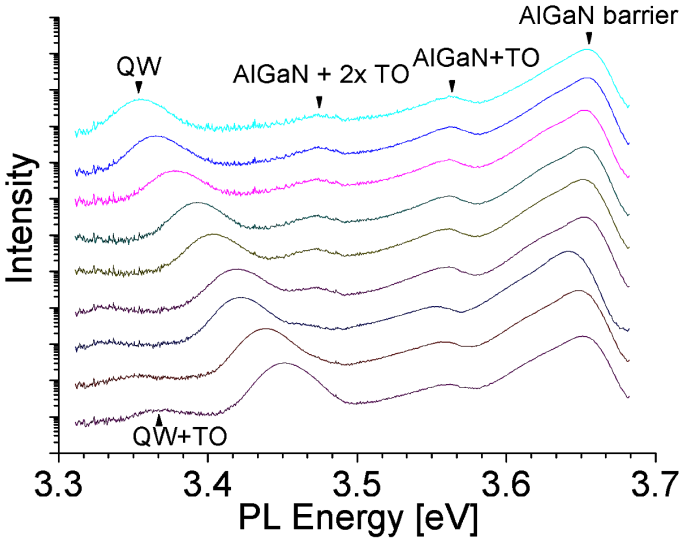

Fig. 5. Raw spectra of the sample, collected for consecutive places on the sample. From right to left one sees $\mathrm{PL}$ of the AlGaN barrier, then its two phonon replicas, $\mathrm{PL}$ of the quantum well (QW), and, for last few series, first phonon replica of the QW PL. Series' labels correspond to the distance from the edge of the sample.

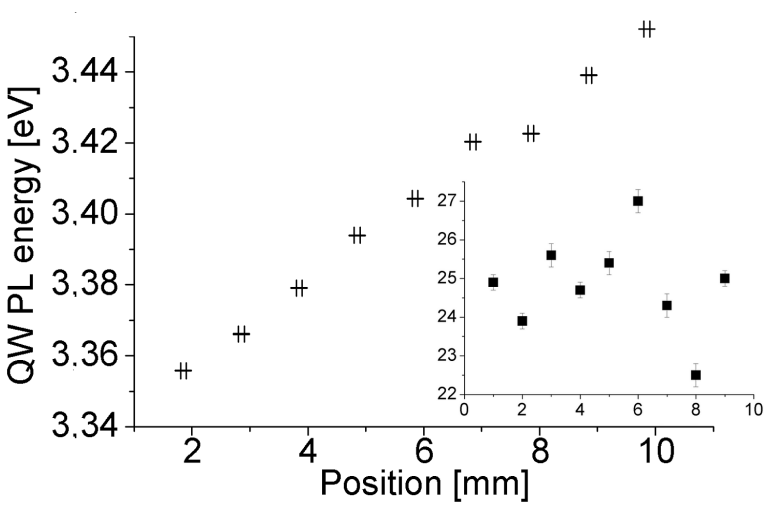

Fig. 6. Energy of the QW PL clearly changes with respect to the position on the sample. The inset provides standard deviations of the peaks with respect to position in $\mathrm{mm}$, whose mean is $24.8 \mathrm{meV}$.

$$
E_{\mathrm{PL}}=E_{\mathrm{e}}+E_{\mathrm{h}}+E_{\mathrm{g}}-E_{\mathrm{exc}}-e F L,
$$

where $E_{\mathrm{g}}$ is energy gap, $E_{\text {exc }}$ is exciton binding energy and $E_{\mathrm{e}}$ and $E_{\mathrm{h}}$ are energies of first quantum levels in well (see Fig. 1) defined as

$$
E_{\mathrm{e}}=\left(\hbar^{2} / 2 m_{\mathrm{e}}^{*}\right)\left[9 \pi m_{\mathrm{e}}^{*} e F /\left(4 \hbar^{2}\right)\right]^{2 / 3},
$$

where $m_{\mathrm{e}}^{*}$ is electron effective mass. $E_{\mathrm{h}}$ is defined analogically. For constant $F, E_{\mathrm{PL}}=E_{0}-e F L$ is linear function of $L$, with constant $E_{0}=E_{\mathrm{e}}+E_{\mathrm{h}}+E_{\mathrm{g}}-E_{\text {exc }}$.

As typical for GaN/AlGaN quantum well system, it is found that luminescence energy is strongly affected by the built-in electric field $F$ and changes linearly with respect to QW width (Fig. 7). This allows for the precise determination of value of $F$ to be $0.66 \mathrm{MV} / \mathrm{cm}$, which is significantly higher than previously reported for studies of $\mathrm{GaN} / \mathrm{Al}_{x} \mathrm{Ga}_{1-x} \mathrm{~N}$ quantum well system [4-7]. 


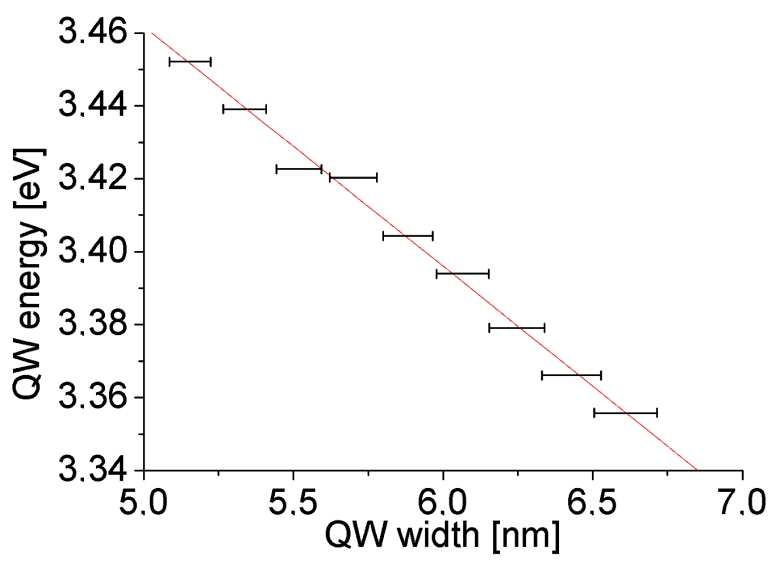

Fig. 7. The correlation of QW PL energy $E$ and the quantum well width $L$ at particular positions on the sample. The fitted linear function of the form $E=$ $E_{0}-e F x\left(E_{0}=3.790(11) \mathrm{eV}\right.$ stands for constant offset as defined in (1), $e$ - elementary charge) implies value of the electric field $F$ of $0.66 \mathrm{MV} / \mathrm{cm}$.

\section{Conclusions}

Using particular properties of the wedge-shaped sample we determine the built-in electric field strength in the GaN/AlGaN quantum well structure. The applied method enables us to make systematic measurement in function of QW width, which allows better control in comparison with previous works that compare results obtained on different samples made in different processes.
Higher value of the electric field strength, as compared to previously reported for similar structures, can be related to the particular properties of the technology used in the MOCVD growth. In particular, understanding of the influence of the strain, that can be different in our case, will need more studies.

\section{References}

[1] O. Ambacher, J. Majewski, C. Miskys, A. Link, M. Hermann, M. Eickho, M. Stutzmann, F. Bernardini, V. Fiorentini, V. Tilak, B. Scha, L.F. Eastman, J. Phys. Condens. Matter 14, 3399 (2002).

[2] K.P. Korona, A. Drabinska, P. Caban, W. Strupinski, J. Appl. Phys. 105, 083712 (2009).

[3] K. Pakula, J.M. Baranowski, J. Borysiuk, Mater. Res. Soc. Symp. Proc. 1040, 165 (2008).

[4] N. Grandjean, B. Damilano, J. Massies, G. Neu, M. Teissere, I. Grzegory, S. Porowski, M. Gallart, P. Lefebvre, B. Gil, M. Albrecht, J. Appl. Phys. 88, 183 (2000).

[5] H.M. Ng, R. Harel, S.N.G. Chu, A.Y. Cho, J. Electron. Mater. 30, 134 (2001).

[6] B. Monemar, H. Haratizadeh, P.P. Paskov, G. Pozina, P.O. Holtz, J.P. Bergman, S. Kamiyama, M. Iwaya, H. Amano, I. Akasaki, Phys. Status Solidi B 237, 353 (2003).

[7] C. Buchheim, R. Goldhahn, G. Gobsch, K. Tonisch, V. Cimalla, F. Niebelschuetz, O. Ambacher, Appl. Phys. Lett. 92, 013510 (2008). 